\title{
La notion de «grave trouble mental»
}

\author{
Philippe Delacrausaza ${ }^{\text {a Nicolas Queloz }}{ }^{\mathrm{b}}$ \\ ${ }^{a}$ Médecin adjoint, responsable du Centre d'expertises, Institut de psychiatrie légale, DP-CHUV, Prilly \\ b Professeur ordinaire de droit pénal et de criminologie, Faculté de droit, Université de Fribourg
}

\section{Der Begriff «psychisch schwer gestört»:}

\section{Überlegungen aus psychiatrischer und strafrechtlicher Sicht}

Für die gerichtliche Anordnung einer strafrechtlichen therapeutischen Massnahme, die darauf abzielt, das Risiko eines erneuten Auftretens von Straftaten zu verringern, ist der Begriff «psychisch schwer gestört» von zentraler Bedeutung. Doch sowohl in psychiatrischer als auch in rechtlicher Hinsicht ist dieser Terminus weiterhin nicht klar definiert. Wahrscheinlich hat unter anderem diese mangelnde Genauigkeit dazu beigetragen, dass seit dem Inkrafttreten des revidierten Strafgesetzbuchs (StGB) im Jahr 2007 eine Zunahme der angeordneten strafrechtlichen therapeutischen Massnahmen zu beobachten ist. Die Autoren setzen sich aus psychiatrischer und rechtlicher Sicht eingehend mit diesem Begriff auseinander und sprechen sich für eine Begriffsklärung aus, um die Zahl der strafrechtlichen therapeutischen Massnahmen zu verringern, die in der Schweiz angeordnet werden.

\section{Introduction}

L'existence d'un «grave trouble mental» liée à la commission d'un crime ou d'un délit est une condition indispensable au prononcé d'une mesure thérapeutique institutionnelle (art. 59 CPS [1]) ou ambulatoire (art. 63 CPS).

La mesure ordonnée lors du jugement a pour objectif de viser une réduction du risque de récidive.

Depuis l'entrée en vigueur de la révision du CPS en 2007, le nombre croissant de détenus faisant en Suisse l'objet d'une mesure thérapeutique institutionnelle [2] suscite toujours plus de questionnement [3]. A-t-on vraiment à faire à plus de délinquants souffrant d'un "grave trouble mental» ou est-ce la perspective sécuritaire qui conduit à un recours accru à cette qualification et à cette mesure?

\section{«Grave trouble mental»: d'une notion juridique indéterminée à une définition psychiatrique très ouverte}

Le «grave trouble mental» est une notion juridique indéterminée, qui renvoie à une classification reconnue, comme la Classification internationale des maladies de l'OMS [4]. Elle signifie que tout l'éventail des phénomènes psychiques anormaux et susceptibles d'être diagnostiqués selon des critères scientifiques peut, en principe, entraîner une mesure thérapeutique.
Le législateur a renoncé sciemment à établir une liste des troubles spécifiques. «Contrairement à l'avis de la commission d'experts, cette mesure pourra également être ordonnée à l'égard d'un délinquant qui souffre de graves troubles de la personnalité» [5], comme c'était déjà le cas de la majeure partie des «délinquants anormaux» traités selon l'ancien Code pénal (art. 43 aCPS). La dépendance aux jeux de hasard, par exemple, est également considérée comme un trouble mental, tout comme la dépendance compulsive à Internet. Toutefois, des troubles mentaux et du comportement liés à l'utilisation de substances psychotropes doivent être examinés sous l'angle de l'art. 60 CPS [6].

Le trouble doit exister au moment de la commission de l'acte comme au moment du jugement. La nécessité d'une mesure de traitement des troubles mentaux doit être constatée par une expertise, conformément à l'art. 56 al. 3 CPS. L'expertise doit être établie par un médecin psychiatre [7]. Dans la pratique, l'expert psychiatre est appelé à se prononcer sur la gravité du trouble diagnostiqué. Cette notion est toutefois malaisée à apprécier, cette dimension n'étant pas clairement définie d'un point de vue psychiatrique.

Pour ce qui est de la notion de trouble, on lit dans le chapitre "Introduction» du manuel de la CIM-10: «Bien qu'il pose problème, le terme 'trouble' est utilisé systématiquement dans la classification. Le recours à d'autres termes, tels que celui d'affection' ou de 'maladie' aurait en effet soulevé des problèmes encore plus importants. Le terme de 'trouble' n'est pas un terme précis: dans la CIM-10, il indique simplement la présence d'un ensemble de symptômes et de comportements cliniquement identifiables, associés, dans la plupart des cas, à un sentiment de détresse et à une perturbation du fonctionnement personnel. Selon la définition donnée ici, une conduite sociale déviante ou conflictuelle, non accompagnée d'une perturbation du fonctionnement personnel, ne doit pas être considérée comme un trouble mental» [8].

La sévérité ou gravité des troubles n'est ni évoquée ni précisée. Dans la littérature scientifique, la notion de trouble mental grave n'est pas plus clairement définie [9]. La notion de trouble mental grave paraît souvent se confondre avec celle de trouble psychotique [10]. En particulier, dans le champ de la psychiatrie forensique, on peut lire: «Nous retenons la définition consensuelle de 
Hodgins des troubles mentaux graves, qui regroupe les diagnostics de schizophrénie, de trouble de l'humeur et de trouble délirant qui correspond dans la nosographie française à la psychose paranoïaque. Cette définition restreinte de la maladie mentale (troubles psychotiques ou dépressifs exclusivement), s'oppose à celle, plus large, du DSM-IV qui inclut, en outre, les troubles de la personnalité, les abus d'alcool et les démences» [11].

Dans les débats parlementaires qui ont mené à l'adoption de la révision du CPS, la référence à la notion de trouble mental grave a été évacuée de la question de la responsabilité pénale, en raison notamment de la difficulté de la préciser. "[...] la commission s'est lancée dans une grande discussion pour savoir ce que recouvre le terme de 'grave trouble mental'. Nous aurions voulu déterminer si ce terme englobe à la fois la maladie psychique, le handicap mental ou le trouble psychologique ou émotionnel passager [...] Mais, finalement, nous nous sommes rendu compte que la responsabilité ou l'irresponsabilité, au moment de commettre un acte, pouvaient être évaluées pour elles-mêmes, avec ou sans diagnostic psychiatrique» [12].

Cette préoccupation n'a cependant pas été reprise pour ce qui concerne l'application des mesures. En fait, depuis 2007, si la notion de "grave trouble mental» a disparu de l'appréciation de la responsabilité pénale (art. 19 CPS), elle a pris une place très forte dans le prononcé des mesures thérapeutiques (art. 59 et 63 CPS).

Et nous constatons finalement que le CPS ne fait du «grave trouble mental» qu'une notion juridique indéterminée, dans l'espoir que la médecine et les psychiatres la définissent et éclairent la lanterne des juristes... Ce qui, dans les faits, ne se réalise pas! Droit et médecine ouvrent ainsi des boîtes ou des boucles qui ne se referment pas.

\section{Jurisprudence et pratique judiciaire}

De la jurisprudence du Tribunal fédéral, nous pouvons relever les éléments suivants:

«[...] toute anomalie mentale au sens médical ne constitue pas une anormalité mentale au sens de la loi, laquelle postule l'existence d'un grave trouble. Elle apporte également la précision qu'un traitement spécial du trouble mental se justifiera uniquement s'il est à prévoir qu'il détournera l'auteur de commettre de nouvelles infractions en relation avec ce trouble» [13].

Doit être considéré comme "grave» le trouble mental qui est significatif sur le plan psychiatrique comme sur le plan juridique. Mais il s'agit d'une notion juridique indéterminée, qui relève du pouvoir d'appréciation du juge et doit être examinée à l'aune du principe de la proportionnalité [14].
«[...] il faut que la structure mentale de l'intéressé s'écarte manifestement de la moyenne par rapport aux autres sujets de droit, mais plus encore par rapport aux autres criminels (message du 21 septembre 1998 concernant la modification du code pénal suisse [...], FF 1999 p. 1812). La référence à la gravité du trouble mental ne correspond pas à une description quantitative du dérangement psychique, mais signifie uniquement que le trouble mental doit être significatif sur le plan psychiatrique comme sur le plan juridique» [15].

«Contrairement au traitement psychiatrique ordonné dans le cadre d'une mesure d'internement (art. 64 al. 4 in fine CPS), la mesure thérapeutique au sens de l'art. 59 CPS vise avant tout 'un impact thérapeutique dynamique', et donc avec une amélioration du pronostic légal, et non la 'simple administration statique et conservatoire' des soins (ATF 137 IV 201 consid. 1.3 p. 204; 134 IV 315 consid. 3.6 p. 323 s.) [...] Pour que la mesure puisse atteindre son but, il faut que l'auteur contribue un minimum au traitement. Il ne faut toutefois pas poser des exigences trop élevées à la disposition minimale de l'intéressé à coopérer à la mesure» [16].

Le risque est grand, dès lors, pour le juge (voire pour le psychiatre) de considérer une pathologie éventuellement présente chez un délinquant comme d'autant plus grave que le crime commis aura été lui-même grave, avec l'inquiétude liée à une réitération élevée. Ce risque pourrait s'accroître dans l'hypothèse où il existe un traitement dynamique, à visée de réduction du risque de récidive.

De plus, si selon le message du Conseil fédéral, «La forme de traitement appropriée ne dépend pas de la gravité du trouble mental: il existe de graves troubles mentaux qui peuvent être traités ambulatoirement alors que des troubles relativement bénins peuvent impliquer un traitement intensif, voire institutionnel, pour permettre à la mesure d'atteindre son objectif» [17], il n'en reste pas moins que dans la pratique, c'est souvent la gravité de l'acte commis qui détermine l'intensité de la mesure (institutionnelle vs ambulatoire).

\section{De l'avis de l'expert à la décision du juge}

Selon les termes de la loi, c'est le juge qui «peut ordonner un traitement» institutionnel (art. 59 CPS) ou ambulatoire (art. 63 CPS) lorsque les conditions du «grave trouble mental» et du risque de récidive [18] sont, selon son appréciation, réalisées.

Pour faire cette appréciation, le juge doit se fonder sur l'expertise d'un médecin psychiatre. Cette expertise doit se déterminer, selon l'art. 56 al. 3 CPS:

«a. sur la nécessité et les chances de succès d'un traitement; 
b. sur la vraisemblance que l'auteur commette d'autres infractions et sur la nature de celles-ci;

c. sur les possibilités de faire exécuter la mesure ainsi que sur le type de mesure préconisé.»

Il est donc intéressant de se demander si, dans leurs jugements, les juges suivent ou s'écartent des propositions faites par les experts psychiatres?

Il faut d'abord rappeler que, selon le Tribunal fédéral, «le juge apprécie en principe librement une expertise et n'est pas lié par les conclusions de l'expert. Toutefois, il ne peut s'en écarter que lorsque des circonstances ou des indices importants et bien établis en ébranlent sérieusement la crédibilité; il est alors tenu de motiver sa décision de ne pas suivre le rapport d'expertise» [19]. Une étude a été menée sur des données d'expertises bâloises réalisées entre 1989 et 2000 [20]. Elle a notamment montré:

- que lorsque les experts recommandaient une mesure thérapeutique ambulatoire $(\mathrm{N}=131)$, les juges ordonnaient cette mesure dans $39 \%$ des cas $(\mathrm{N}=51)$, ne prononçaient aucune mesure dans $56 \%$ des cas $(\mathrm{N}=73)$ et ordonnaient une mesure thérapeutique institutionnelle (plus contraignante) dans $2 \%$ des cas $(\mathrm{N}=3)$;

- et que lorsque les experts recommandaient une mesure thérapeutique institutionnelle $(\mathrm{N}=51)$, les juges ordonnaient cette mesure dans $31,5 \%$ des cas ( $\mathrm{N}=16$ ), ne prononçaient aucune mesure dans 53\% des cas $(\mathrm{N}=27)$, ordonnaient une mesure thérapeutique ambulatoire (moins contraignante) dans $14 \%$ des cas $(\mathrm{N}=7)$ et une mesure d'internement (plus contraignante) dans $2 \%$ des cas $(\mathrm{N}=1)$.

Ces résultats tendent à démontrer une grande indépendance et «souveraineté» des juges par rapport aux avis et propositions des experts, pour la période considérée. Mais depuis lors, le cadre légal a changé, les experts forensiques se sont professionnalisés et spécialisés, et les juges sont devenus extrêmement (voire excessivement) attentifs aux aspects sécuritaires de leurs jugements.

Il serait utile de mener un même type d'étude sur les années récentes (2000 à 2015), car nous pouvons faire l'hypothèse qu'aujourd'hui, d'une part les juges, ainsi que les experts, se montrent plus «prudents» ou restrictifs et, d'autre part, que les juges se montrent moins indépendants ou "souverains» et que leurs jugements avalisent plus fréquemment les recommandations des experts.
Nous faisons également l'hypothèse que, dans ce contexte, une définition plus précise (et plus restrictive) de la notion de trouble mental grave pourrait permettre une réduction du nombre de mesures ordonnées.

\section{Disclosure statement}

Aucun conflit d'intérêts.

\section{Références}

1 CPS: code pénal suisse (du 21.12.1937: www.admin.ch/opc/fr/ classified-compilation/19370083/index.html).

2 De 2007 (84) à 2013 (120), il y a eu une augmentation de 43\% des prononcés de l'art. 59 CPS (avec un pic à 135 cas en 2010).

3 Sur les difficultés posées par l'art. 59 CPS, voir notamment: Brägger B. Massnahmenvollzug an psychisch kranken Straftätern in der Schweiz: Eine kritische Auslegeordnung, Schweizerische Zeitschrift für Kriminologie-Revue Suisse de Criminologie, 2014, 2 36-44, et Queloz N. Risques de mauvais traitement en raison de situations de vulnérabilité dans l'exécution des sanctions pénales, in Queloz N, Noll Th, von Mandach L, Delgrande N (Eds.) Verletzlichkeit und Risiko im Justizvollzug - Vulnérabilité et risques dans l'exécution des sanctions pénales, Berne, Stämpfli, 2015, 1-17.

4 CIM-10, Classification internationale des troubles mentaux et des troubles du comportement; OMS (1993) Ed. Masson.

5 Message du Conseil fédéral du 21.09.1998, FF 19991882.

6 Art. 60 CPS: Traitement institutionnel des addictions. Dans la classification du DSM-5, la dépendance aux jeux de hasard figure précisément dans la catégorie des «substance-related and other addictive disorders".

7 ATF 140 IV 49 (et aussi 6B_850/2013 pour l'expertise relative à la prolongation d'une mesure).

8 CIM-10, Classification internationale des troubles mentaux et des troubles du comportement; OMS (1993) Ed. Masson, p. 4.

9 An empirical literature review of definitions of severe and persistant mental illness. A. P. Schinnar et al. Am J Psychiatry 1990; 147:1602-8).

10 La divulgation du trouble mental et les mesures d'accommodements de travail: deux facteurs du maintien en emploi des personnes aux prises avec un trouble mental grave. M. Corbière et al. L'Encéphale (2014) 40 S91-S102.

11 Homicide et maladie mentale grave: quelles sont les différences sociodémographiques, cliniques et criminologiques entre des meurtriers malades mentaux graves et ceux indemnes de trouble psychiatriques? S. Richard-Devantoy et al. L'Encéphale (2009) 35, 304-14.

12 AB 2001 N 544 / BO 2001 N 544 (www.parlament.ch/ab/frameset/ f/n/4608/30381/f_n_4608_30381_30428.htm).

13 Arrêt 6B 457/2007 du 12.11.2007, 5.1.

14 Arrêt $6 \mathrm{~S} .427 / 2005$ du 6.04.2006, considérant 2.3.

15 Arrêt 6B_77/2012 du 18.06.2012, considérant 2.1.2; et arrêt 6B 372/2012 du 27.09.2012, considérant 2.1.

16 Arrêt 6B_763/2014 du 6.01.2015, considérant 3.1.2.

17 Message du Conseil fédéral du 21.09.1998, FF 19991883.

18 Lorsqu'il est à prévoir, selon le CPS, que le traitement détournera l'auteur «de nouvelles infractions en relation avec ce trouble».

19 ATF 133 II 384, considérant 4.2.3; et arrêt 6B_77/2012 du 18.06.2012 21.3

20 Hachtel H, Aenis L, Sahin D, Graf M. Der Einfluss des Sachverständigengutachtens auf das Urteil aus psychiatrischer und juristischer Sicht, Schweizerische Zeitschrift für KriminologieRevue Suisse de Criminologie, 2014, 2, 3-9. 